IZA DP No. 9022

Changing Income Inequality and Panel Income Changes

Robert Duval Hernández

Gary S. Fields

George H. Jakubson

April 2015 


\title{
Changing Income Inequality and Panel Income Changes
}

\author{
Robert Duval Hernández \\ University of Cyprus \\ and CIDE \\ Gary S. Fields \\ Cornell University \\ and IZA \\ George H. Jakubson \\ Cornell University
}

Discussion Paper No. 9022
April 2015

IZA

P.O. Box 7240

53072 Bonn

Germany

Phone: +49-228-3894-0

Fax: +49-228-3894-180

E-mail: iza@iza.org

Any opinions expressed here are those of the author(s) and not those of IZA. Research published in this series may include views on policy, but the institute itself takes no institutional policy positions. The IZA research network is committed to the IZA Guiding Principles of Research Integrity.

The Institute for the Study of Labor (IZA) in Bonn is a local and virtual international research center and a place of communication between science, politics and business. IZA is an independent nonprofit organization supported by Deutsche Post Foundation. The center is associated with the University of Bonn and offers a stimulating research environment through its international network, workshops and conferences, data service, project support, research visits and doctoral program. IZA engages in (i) original and internationally competitive research in all fields of labor economics, (ii) development of policy concepts, and (iii) dissemination of research results and concepts to the interested public.

IZA Discussion Papers often represent preliminary work and are circulated to encourage discussion. Citation of such a paper should account for its provisional character. A revised version may be available directly from the author. 
IZA Discussion Paper No. 9022

April 2015

\section{ABSTRACT}

\section{Changing Income Inequality and Panel Income Changes}

When economic growth (or economic decline) takes place, who benefits and who is hurt how much? The more traditional way of answering this question is to compare two or more comparable cross sections and gauge changing income inequality among countries or individuals. A newer way is to utilize data on a panel of countries or a panel of people and assess the pattern of panel income changes. How do these two approaches relate to one another? This paper shows, first, that it is possible to have all four combinations - rising or falling inequality and divergent or convergent panel income changes, and second, under what conditions, for various measures of rising/falling inequality and various measures of divergent/convergent income changes, each of the four possible combinations can arise.

JEL Classification: J31, D63

Keywords: income inequality, economic mobility

Corresponding author:

Gary S. Fields

Cornell University

270 Ives Hall

Ithaca, NY 14853-3901

USA

E-mail: gsf2@cornell.edu 


\section{Introduction}

Who benefits and who is hurt how much when an economy grows or contracts? The more traditional way of answering this question is to compare data from two or more cross sections and gauge changing income inequality among individuals or households.

A newer way of gauging who benefits and who is hurt is to utilize data on a panel of people and assess the pattern of panel income changes.

A fundamental difference between these two approaches is that the income inequality approach treats people anonymously, while the panel data approach works with the income changes of identified people. More specifically, when looking at income inequality using such familiar tools as Lorenz curves and inequality indices, the analyst looks at the income of whoever is in the p'th position in each distribution regardless of whether that is the same person in one distribution as in the other. By contrast, when looking at panel income changes, the analyst first identifies which individual is in the p'th position in the initial distribution and follows that person over time, even if that person is in a different position later on.

Thus, a statement about the persons in a particular group $g$ say, the richest $1 \%$ or poorest $10 \%$ - means different things in the two approaches. The standard inequality analysis permits statements of the type "the anonymous richest $1 \%$ got richer while the anonymous poorest $10 \%$ got poorer" while the panel data analysis makes a different type of statement: "those who started in the richest $1 \%$ experienced income changes of such and such amount while those who started in the poorest $10 \%$ experienced income changes of a different amount." Both approaches provide true information, but to the extent that people move around within the income distribution, it is not the same information.

Empirical studies have shown that in about half the cases income inequality has increased, and in about half, inequality has decreased (Atkinson and Bourguignon, 2000, 2015). On the other hand, panel data studies show, almost without exception, that regardless of whether income inequality is rising or falling, the panel income changes are on average most positive for the initially lowest income people and least positive (or most negative) for the initially highest income people; these studies are reviewed by Fields (2010) for panel income changes among individuals, by Solon (1999) for differences in incomes between parents and their children, and by Durlauf and Quah (1999) and Korotayev et al. (2011) for changes in the mean incomes of coun- 
tries.

The idea that a pattern of panel changes whereby those at the bottom gain more than those at the top necessarily results in falling inequality was first raised by Francis Galton in 1886. Later scholars demonstrated that no such implication holds, and Galton's assertion has come to be dubbed "Galton's fallacy" (see, for example, Bliss, 1999).

The literature also offers a claim regarding the opposite set of circumstances. Consider a panel of countries with per capita incomes in comparable currency units - Purchasing Power Parity-adjusted dollars, for example. Define $\beta$-divergence (convergence) as arising when a regression of final logincome on initial log-income produces a regression coefficient greater than (less than) one. Define $\sigma$-convergence (divergence) as arising when the variance of log-incomes falls (rises) from the initial year to the final year. It is proven in the literature that $\beta$-divergence measured in this way and $\sigma$ convergence measured in this way cannot arise simultaneously - more specifically, $\sigma$-convergence implies $\beta$-convergence, but $\beta$-convergence does not imply $\sigma$-convergence (Furceri, 2005; Wodon and Yitzhaki, 2006).

Is it possible to have convergent panel income changes, that is, the income changes we see following named individuals over time, and rising income inequality? Is it possible to have divergent panel income changes and falling income inequality? Are the possibilities in times of economic growth different from those in times of economic decline? One purpose of this paper is to derive what is possible and what is impossible. Contrary to the suggestions in the preceding paragraphs, we show that it is indeed possible to have rising or falling inequality along with convergent or divergent mobility, both in times of economic growth and in times of economic decline (see Table 1).

The second purpose of this paper is to derive conditions under which, for various measures of rising/falling inequality and various measures of convergent/divergent income changes, each of the four possibilities can arise. A number of propositions are derived.

Overall, the results in this paper reaffirm what has been known in the literature for some time. Whether income inequality rises or falls in the cross section is one thing. Whether panel income changes are divergent or convergent is another thing. Rising/falling inequality and divergent/convergent income changes are both interesting; they are, however, different.

But the results here are not just a reaffirmation. This paper goes beyond the previous literature in deriving precise conditions under which i) income inequality rises or falls, ii) panel income changes are divergent or convergent, 


\section{Table 1: Possibilities for Rising/Falling Inequality and Convergent/Divergent Panel Income Changes}

\begin{tabular}{|c|c|c|}
\hline & Rising Inequality & Falling Inequality \\
\hline Convergent & $\checkmark$ & $\checkmark$ \\
Panel & & \\
Income & & \\
Changes & & $\checkmark$ \\
\hline Divergent & $\checkmark$ & \\
Panel & & \\
Income & & \\
Changes & & \\
\hline
\end{tabular}

$\checkmark$ : This cell is possible both in times of economic growth and in times of economic decline.

iii) the four possibilities in Table 1 can arise, and iv) when certain combinations cannot arise for particular measures of changing inequality and convergence/divergence. These conditions are derived in Section 3, catalogued in Table 5, and summarized in Section 4.

\section{Measurement Issues and Illustrative Exam- ples}

The two key variables in this research are income inequality and panel income changes. "Income" is the term used for the economic variable of interest, which could be total income, labor earnings, consumption, or something else. The income recipient will be called a "person", but the results apply equally to households, workers, per capitas, or adult equivalents.

\subsection{Income Inequality}

When is income inequality rising or falling? This question is answered by using a functional or an index to represent the inequality at two points in time and then to compare them.

Income inequality and the change in income inequality are conceptualized and measured in a number of ways. "Relative inequality" is concerned with 
income comparisons measured in terms of ratios, "absolute inequality" with income comparisons measured in terms of dollar differences.

A widely-used criterion for determining which of two income distributions is relatively more equal than another is the three-part Lorenz criterion, which states i) if Lorenz curve A lies somewhere above and never below Lorenz curve B, A is more equal than B, ii) if Lorenz curves A and B coincide, then $\mathrm{A}$ and $\mathrm{B}$ are equally unequal, and iii) if the Lorenz curves of $\mathrm{A}$ and $\mathrm{B}$ cross, the relative inequalities of $\mathrm{A}$ and $\mathrm{B}$ cannot be compared using the Lorenz criterion alone. Judging a Lorenz-dominant distribution to be more equal than a Lorenz-dominated one is equivalent to making inequality comparisons on the basis of four commonly-accepted relative inequality axioms: anonymity, scale-independence, population-independence, and the transfer principle (Fields and Fei, 1978). ${ }^{1}$

Yet, despite its appeal, the Lorenz criterion is not universally used for two reasons: its ordinality and its incompleteness. When the Lorenz criterion does render a verdict about which of two income distributions is more equal than another, it can only say that $\mathrm{A}$ is more equal than $\mathrm{B}$ but not how much more equal A is than B. And when Lorenz curves cross, the Lorenz criterion cannot render a verdict.

Those analysts who seek a complete cardinal comparison of the inequalities of two income distributions are led to use one or more inequality indices. For present purposes, these indices can be put into three categories:

1. Lorenz-consistent relative inequality indices: An inequality index is Lorenz-consistent if, when one Lorenz curve dominates another, the index registers the dominant distribution as (weakly) more equal. A partial listing of Lorenz-consistent relative inequality indices includes the Gini coefficient, income share of the richest X\%, income share of the poorest Y\%, Atkinson index, Theil index, the decile ratios, and the coefficient of variation and its square. For details, see Sen (1997) and Cowell (2011).

2. Lorenz-inconsistent relative inequality indices: An inequality index is Lorenz-inconsistent if, when one Lorenz curve dominates another, it is ever the case that the index shows the Lorenz-dominant distribution to

\footnotetext{
${ }^{1} \mathrm{~A}$ similar comparison can be made for the measurement of absolute inequality if we replace the axiom of scale-independence, by one of translation-invariance, and work instead with what is known as an absolute Lorenz Curve (see below).
} 
be less equal. One commonly-used relative inequality index is Lorenzinconsistent: the variance of the logarithms of income. This index violates the transfer principle - that is, it is possible to make a rankpreserving transfer of income from a relatively rich person to a relative poorer person and yet the index can register an increase in relative inequality (Foster and Ok, 1999; Cowell, 2011).

3. Lorenz-inconsistent absolute inequality indices: All absolute inequality indices are Lorenz-inconsistent because they violate the axiom of scaleindependence. For example, the variance of incomes is not Lorenzconsistent: doubling everyone's income increases inequality by a factor of four.

In our work below, we emphasize Lorenz curve comparisons and Lorenzconsistent inequality indices. However, we also present results pertaining to the measurement of absolute inequality, and we give attention to the variance of incomes and the variance of log-incomes despite their Lorenz-inconsistency, in part because the literature has done so and in part because results can be gotten using them.

\subsection{Divergent and Convergent Panel Income Changes}

By definition, income mobility analysis entails looking at the joint distribution of incomes at two or more points in time. This is an analysis of panel income changes since we follow a particular individual. Our analysis in this paper is limited to income changes between an initial period and a final period.

The income mobility literature distinguishes six mobility concepts: timeindependence, positional movement, share movement, directional income movement, non-directional income movement, and mobility as an equalizer of longer-term incomes relative to initial (Fields, 2008). For purposes of characterizing the pattern of panel income changes in this paper, the relevant concept is directional income movement among panel people - that is, who gains or loses how much, from an initial date to a final one.

Panel income changes are said to be divergent when the income recipients who started ahead on average get ahead faster than those who started behind. It is convergent when those who started ahead on average get ahead more slowly than those who started behind. It is neutral when neither is the case. 
What it means to get ahead at a faster, slower, or same rate itself requires careful specification. In the macroeconomics literature, the object of interest is nearly always the growth rate in percentages, often approximated by changes in log-income (see, for example, Barro, 1991; Sala-i-Martin, 1996). On the other hand, the literature on panel income changes among individuals or households presents a more varied picture; some studies use income changes in dollars, while others use changes in log-dollars, percentage changes, changes in income shares, or changes in income quantiles such as deciles or centiles (Jäntti and Jenkins, 2015).

Much of the literature assesses divergence or convergence by assuming a linear relationship between final income and initial income or between income change and initial income. Much but not all of our analysis works with the linearity assumption as well.

Accordingly, we gauge divergence or convergence as follows. Consider a generic income variable $y$, which might be dollars, log-dollars, or income shares. We can have the levels-on-levels regression $y_{1}=\alpha_{y}+\beta_{y} y_{0}+u_{y}$ or the change-on-initial regression $\Delta y \equiv y_{1}-y_{0}=\gamma_{y}+\delta_{y} y_{0}+u_{y}$. The two regressions are linked by the relationship $\delta_{y}=\beta_{y}-1$. Divergence is said to arise when $\beta_{y}>1$, or equivalently, when $\delta_{y}>0$. Likewise, we have convergence when $\beta_{y}<1 \Longleftrightarrow \delta_{y}<0$. We may be interested in divergence or convergence of true proportional changes (as opposed to the logarithmic approximation thereof), in which case we would want to regress the proportional change in dollars on initial dollars: pch $\mathrm{d} \equiv\left(d_{1}-d_{0}\right) / d_{0}=\phi+\theta d_{0}+u_{p c h}$. Proportional changes are divergent or convergent according to whether $\theta$ is greater or less than zero.

\subsection{A Matrix of Possibilities}

In what came before, we identified three ways of determining the direction of change in relative inequality - i) Lorenz-improvement and Lorenz-worsening, ii) Change in a Lorenz-consistent relative inequality index, and iii) Change in Lorenz-inconsistent relative inequality measures - and four ways of assessing divergence or convergence: i) Dollar changes, ii) Share changes, iii) Log-dollar changes, and iv) Proportional changes.

Can each possible combination of rising or falling relative inequality and divergent or convergent panel income changes arise? The answer is yes, provided they are measured suitably. Table 2 displays examples for each of the possible combinations. 
Table 2: Examples of Possibilities in Times of Economic Growth and Decline.

Final on Initial Regression: $y_{1}=\alpha_{y}+\beta_{y} y_{0}+u_{y}$

Changes Regression: $\Delta y_{1}=\gamma_{y}+\delta_{y} y_{0}+u_{y}$

Proportional Changes Regression: $\frac{d_{1}-d_{0}}{d_{0}}=\phi+\theta d_{0}+u_{p c h}$

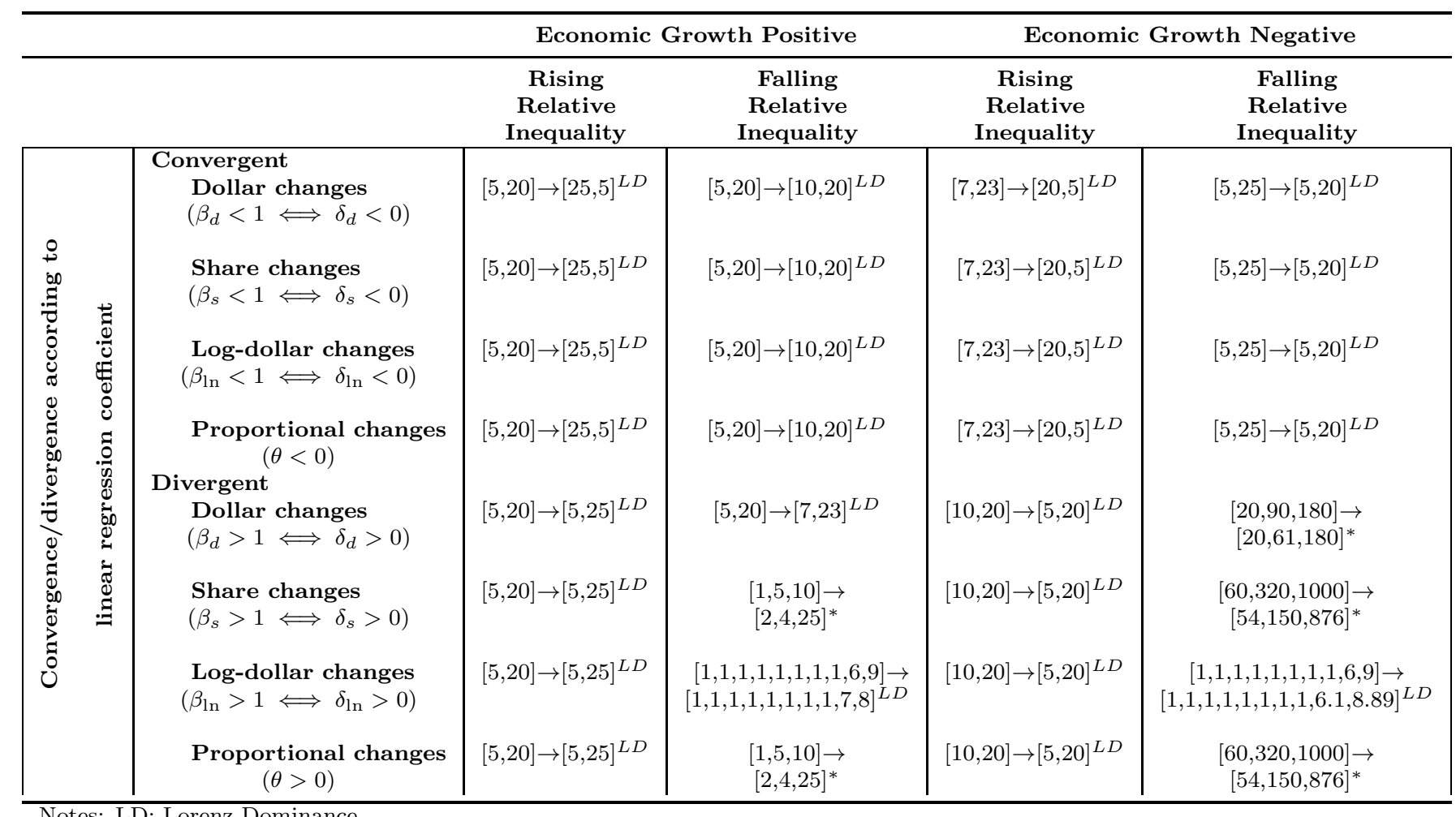

*: If measure changing inequality by income share of the poorest tercile, because Lorenz curves cross. 
To demonstrate the possibilities of most of the combinations, just two people are needed. But to get the remaining combinations, we need to complicate the examples by adding more people and choosing our measures carefully.

Examples prove possibilities; they do not produce exact conditions. In the next section, we derive a number of necessary and sufficient conditions for the various possibilities.

\section{Mathematical Results}

In this section we analytically develop a set of results that establish the connection between changes in relative inequality and our several income change concepts. First, we establish some common notation and definitions, then in sections 3.2-3.4 we derive conditions on possibilities and impossibilities for different ways of measuring inequality and income changes. In everything that follows we consider regressions done on population and abstract from all issues of inference.

\subsection{Notation and Definitions}

Consider an economy with $\mathrm{n}$ individuals observed over two time periods, initial (or 0), and final (or 1).

Denote by $d_{i t}$ the income of individual i in period t measured in constant monetary units (e.g., real dollars). Whenever possible we will avoid expressing the individual subindex i to avoid clutter. Vectors are denoted by bold fonts, e.g., $\mathbf{d}_{\mathbf{t}}=\left(d_{1 t}, d_{2 t}, \ldots, d_{n t}\right)^{\prime}$.

The basic building block of panel data analysis is the data matrix $\mathbf{D}$,

$$
\mathbf{D}=\left[\begin{array}{ll}
\mathbf{d}_{\mathbf{0}} & \mathbf{d}_{\mathbf{1}}
\end{array}\right]=\left[\begin{array}{cc}
d_{10} & d_{11} \\
d_{20} & d_{21} \\
\vdots & \vdots \\
d_{n 0} & d_{n 1}
\end{array}\right] \quad \text { in which } d_{10} \leq d_{20} \leq \ldots \leq d_{n 0}
$$

Each row of the $\mathbf{D}$ matrix includes the incomes of individual $i$ in the initial year $d_{i 0}$ and final year $d_{i 1}$. These rows are sorted in ascending order of initial income.

Let each column of $\mathbf{D}$ be transformed by dividing the incomes by mean income in that year $\mu_{t}$. The resulting share matrix can be written as $\mathbf{S}=$ $\left[\begin{array}{ll}\mathbf{s}_{\mathbf{0}} & \mathbf{s}_{\mathbf{1}}\end{array}\right]$. 
In addition to income shares, we will also deal with other strict monotonic transformations of income, like log-incomes, $\ln d$.

More generically, when a result can be derived both for income in dollars and for a transformation of it, we will denote by $y_{t}=f\left(d_{t}\right)$ the income variable transformed by the strictly monotonically increasing function $f(\cdot){ }^{2}$

For the most part, income vectors and their transformations are sorted in ascending order of individuals' initial-period incomes. An exception to this is the final income-share vector $\mathbf{s}_{\mathbf{c}}$, where the sorting is ascending in final-period income; such sorting is important for Lorenz curve calculations.

\section{Definition. Vector of Final Shares in Ascending Order.}

Let $P(\cdot)$ be a permutation operator. Then, define $\mathbf{s}_{\mathbf{c}}=\left(s_{1 c}, \ldots, s_{n c}\right)$ as the final income-share vector when incomes are sorted in ascending order of final income, i.e.

$$
\mathbf{s}_{\mathbf{c}} \equiv P\left(\mathbf{s}_{\mathbf{1}}\right) \quad \text { such that } \quad s_{i c} \leq s_{j c} \forall i \leq j .
$$

With this notation we can now define the Lorenz Dominance criterion.

\section{Definition. Lorenz Dominance.}

Let $s_{j 0}$ be the initial income-share of the individual in position $\mathrm{j}$, when shares are sorted in ascending order of initial income. Let $s_{j c}$ be the final incomeshare of the individual in position $\mathrm{j}$, when shares are sorted in ascending order of final income. The final income distribution Lorenz-dominates the initial one whenever

$$
\begin{array}{ll}
s_{1 c}+s_{2 c}+\ldots+s_{j c} \geq s_{10}+s_{20}+\ldots+s_{j 0} & \text { for } \mathrm{j}=1,2, \ldots, \mathrm{n}-1 \text { and } \\
s_{1 c}+s_{2 c}+\ldots+s_{j c}>s_{10}+s_{20}+\ldots+s_{j 0} & \text { for some } \mathrm{j}<\mathrm{n} .
\end{array}
$$

In other words, having the final period distribution Lorenz-dominate the initial one means that the final distribution is more equally distributed than the initial one according to this criterion. This situation is sometimes also referred as a "Lorenz-improvement" when going from $\mathbf{d}_{\mathbf{0}}$ to $\mathbf{d}_{\mathbf{1}}$. Similarly, if the previous inequalities are reversed we talk of a "Lorenz-worsening". 3

\footnotetext{
${ }^{2}$ Since the identity function $\mathrm{x}=\mathrm{f}(\mathrm{x})$ is a strictly increasing monotonic function too, $y$ can include income in dollars as well.

${ }^{3}$ The literature usually expresses condition (2) using income as a share of total income. In order to make an easier link with the regressions involving share changes we express it in terms of shares of mean income. It is obvious that the Lorenz curves are the same in the two cases, and hence the inequality comparisons using the Lorenz criteria are also the same.
} 
Following standard notation, we will denote the Lorenz Curve of income in period t by $L C_{t}$, and $L C_{1} \succ L C_{0}$ means that the Lorenz curve in period 1 dominates that of period 0 , namely incomes in period 1 are more equally distributed than the ones in period 0 according to the Lorenz-criterion. If the domination is weak we denote it as $L C_{1} \succeq L C_{0}$, which means that incomes in period 1 are at least as equally distributed as those in period 0 by the Lorenz criterion. $I(\cdot)$ will be used to denote an arbitrary relative inequality measure.

Another concept that we will need throughout the paper is that of a Rank-Preserving Transfer, defined next.

Definition. Equalizing Rank-Preserving Transfer.

A rank-preserving equalizing transfer $h>0$ is a transfer of income between two individuals with ranks $i$ and $j$ for $i>j$, such that:

$$
\begin{array}{llrl}
d_{k 0} & =d_{k 1} & & \text { for } k \neq i, j, \\
d_{i 1} & =d_{i 0}-h, & & \\
d_{j 1} & =d_{j 0}+h, & & \text { where: } \\
\text { if } i & =j+1, & & h<\left(d_{i 0}-d_{j 0}\right) / 2 ; \\
\text { if } i & >j+1, & & h<\min \left[\left(d_{j+1,0}-d_{j 0}\right),\left(d_{i 0}-d_{i-1,0}\right)\right] .
\end{array}
$$

A rank-preserving disequalizing transfer is defined similarly.

Finally, recall the definitions of divergence and convergence and the accompanying notation. For a generic income variable y, which might be dollars, log-dollars, or income shares, we can have the levels-on-levels regression $y_{1}=\alpha_{y}+\beta_{y} y_{0}+u_{y}$ or the change-on-initial regression $\Delta y \equiv y_{1}-y_{0}=$ $\gamma_{y}+\delta_{y} y_{0}+u_{y}$. These two regressions are linked by the relationship $\delta_{y}=\beta_{y}-1$. Divergence is said to arise when $\beta_{y}>1$, or equivalently, when $\delta_{y}>0$. Likewise, we have convergence when $\beta_{y}<1 \Longleftrightarrow \delta_{y}<0$. Alternatively, we can regress the exact proportional change in dollars on initial dollars: pch $\mathrm{d} \equiv\left(d_{1}-d_{0}\right) / d_{0}=\phi+\theta d_{0}+u_{p c h}$. Proportional changes are divergent or convergent according to whether $\theta$ is greater or less than zero.

\subsection{Lorenz Dominance and Income Changes}

We begin by presenting results on the relationship between income changes and changes in inequality as gauged by the Lorenz Dominance criterion. As mentioned in section 2, this criterion is the most accepted way of judging whether relative inequality has risen or fallen. 


\subsubsection{Lorenz Dominance and Share Changes}

Since the Lorenz criterion (2) is formulated in terms of income shares, the natural way to link it with a change regression like the ones used in mobility studies is to compare it to a regression also expressed in shares. In particular, in this section we derive a connection between the Lorenz Dominance criterion (2) and a share-change regression

$$
\Delta s \equiv s_{1}-s_{0}=\gamma_{s}+\delta_{s} s_{0}+u_{s} .
$$

Both equations (2) and (3) involve initial and final income-shares. However, the final period shares appear sorted differently in the two expressions. More specifically, in condition (2), final shares $\mathbf{s}_{\mathbf{c}}$ are sorted in ascending of order of final shares, while in equation (3) final shares $\mathbf{s}_{\mathbf{1}}$ are sorted in ascending order of initial shares.

It is easy to show that the sign of the coefficient $\delta_{s}$ in regression (3) is determined by the sign of the covariance

$$
\operatorname{cov}\left(\Delta s, s_{0}\right)=\frac{\sum_{i}\left(s_{i 1}-s_{i 0}\right) s_{i 0}}{n} .
$$

Using vector $\mathbf{s}_{\mathbf{c}}$ as defined in (1), we can decompose this covariance as

$$
\operatorname{cov}\left(\Delta s, s_{0}\right)=\frac{\sum_{i}\left[\left(s_{i 1}-s_{i c}\right)+\left(s_{i c}-s_{i 0}\right)\right] s_{i 0}}{n} .
$$

That is, whether share changes are convergent or divergent is determined by the sum of two terms:

$$
\begin{aligned}
& W=\frac{\sum_{i}\left(s_{i c}-s_{i 0}\right) s_{i 0}}{n} \\
& X=\frac{\sum_{i}\left(s_{i 1}-s_{i c}\right) s_{i 0}}{n} .
\end{aligned}
$$

$W$ captures the component of the covariance associated with changes in the shape of the income distribution if positions remain unchanged, and $X$ captures the component of the covariance associated with positional change, under a fixed marginal distribution. ${ }^{5}$ These are sometimes called "structural mobility" and "exchange mobility", respectively.

We can derive the following two key Lemmas for these terms.

\footnotetext{
${ }^{4}$ Recall average share changes are zero by construction.

${ }^{5}$ This is so because if positions were to remain unchanged $s_{c}-s_{0}$ would be the share change due to a change in the shape of the distribution.
} 
Lemma 1. Let $W$ be given by equation (4), then:

i) A Lorenz-improvement $\left(L C_{1} \succ L C_{0}\right)$ implies $W<0$.

ii) A Lorenz-worsening $\left(L C_{1} \prec L C_{0}\right)$ implies $W>0$.

Proof: See Appendix.

In other words, in the absence of Lorenz-crossings, the sign of $W$ fully reflects whether there has been a fall or a rise in inequality judged by the Lorenz-criterion.

As previously mentioned, when looking at income changes we care not only about how the distribution of income evolves, but also about who moved to a different position across periods. This is reflected by the transition from $\mathbf{s}_{\mathbf{c}}$ to $\mathbf{s}_{\mathbf{1}}$. In this transition, share changes will be convergent, since in the reranking of individuals there will always be a positive transfer of income shares from a relatively richer individual to a poorer one.

Lemma 2. Let $X$ be given by equation (4), then $X \leq 0$.

Proof: See Appendix.

With these two results we can proceed to analyze the connection between share mobility and changes in inequality as measured by Lorenz comparisons. For simplicity, let us begin by analyzing the case when there are no positional changes between initial and final periods.

\section{The case of no change in positions}

If all individuals keep their same rank in the initial and final distribution, this is called zero positional change, or synonymously, zero positional mobility. In this case, vector $\mathbf{s}_{\mathbf{c}}$ will equal the final share vector $\mathbf{s}_{\mathbf{1}}$, and the sign of $\delta_{s}$ is determined exclusively by $W$. Given Lemma 1 and the connection between $W$ and $\delta_{s}$, in the absence of positional changes, the next Proposition follows immediately.

Proposition 1. Lorenz Dominance and Convergent/Divergent Share Changes Without Positional Change.

Suppose that when the income vector goes from $\mathbf{d}_{\mathbf{0}}$ to $\mathbf{d}_{\mathbf{1}}$, the transition involves no change in positions. Then:

i) A Lorenz-improvement $\left(L C_{1} \succ L C_{0}\right)$ implies convergence in shares $\left(\delta_{s}<\right.$ $0)$ 
ii) A Lorenz-worsening $\left(L C_{1} \prec L C_{0}\right)$ implies divergence in shares $\left(\delta_{s}>0\right)$.

Proof: See Appendix.

The intuition (and proof) behind this proposition is related to a wellknown result in the inequality literature stating that a disequalization in the Lorenz sense can be achieved by a series of income transfers from poorer to richer individuals that keep unaltered the individual ranks between the initial and the final periods (see for instance Fields and Fei, 1978). These transfers generate by construction divergent share changes. The exact opposite occurs when there is a Lorenz improvement (i.e. a fall in inequality) and positions remain unchanged.

In other words, as long as we restrict ourselves to the case of no positional mobility and no crossings of Lorenz curves, share mobility and changes in inequality fully align, in the sense that rising inequality only occurs with divergent share-changes and falling inequality only occurs with convergent share-changes.

\section{The case of positional changes}

Once we allow for positional changes we need to consider not only the transition from $\mathbf{s}_{\mathbf{0}}$ to $\mathbf{s}_{\mathbf{c}}$, but also from $\mathbf{s}_{\mathbf{c}}$ to $\mathbf{s}_{\mathbf{1}}$. In this last step the shape of the income distribution remains unchanged and pairs of individuals swap incomes and therefore positions.

In other words, when going from $\mathbf{s}_{\mathbf{0}}$ to $\mathbf{s}_{\mathbf{1}}$ in the presence of positional changes (but not of Lorenz-crossings) there are two forces at play. The first one, change in the distribution shape with fixed positions, can lead to convergent or divergent share changes, depending on whether there is equalization or disequalization of the anonymous income distribution. The second one, positional rearrangement with a fixed marginal distribution, leads to convergent share-changes always.

In the case of a Lorenz-improvement both components go in the same direction, and share changes are convergent. However, if the income distribution becomes more unequal by the Lorenz-criterion, the two components will move in opposite directions, and depending on which force is dominant there will be convergence or divergence in shares as measured by $\delta_{s}$ in equation (3).

Proposition 2 gives the relation between Lorenz-dominance and share income changes, while Table 3 gives the precise conditions under which each 
combination of convergent/divergent share changes can occur under Lorenzdominance. ${ }^{6}$

\section{Proposition 2. Lorenz Dominance and Convergent/Divergent Share Changes With Positional Change}

i) A Lorenz-improvement $\left(L C_{1} \succ L C_{0}\right)$ implies share convergence $\left(\delta_{s}<0\right)$.

ii) If share changes are non-convergent $\left(\delta_{s} \geq 0\right)$ then either a weak Lorenzworsening has taken place $L C_{0} \succeq L C_{1}$, or the Lorenz curves of incomes in periods 0 and 1 cross.

Proof: See Appendix.

When share changes are divergent, the share of the rich grows relative to that of the poor. This should lead to disequalization. Hence by part ii) of the Proposition, the only possible way to register a fall in inequality in this instance is for Lorenz curves to cross. ${ }^{7}$

\section{Table 3: Conditions for Convergent/Divergent Share Changes under Lorenz Dominance}

\begin{tabular}{|c|c|c|}
\hline & Lorenz-Worsening & Lorenz-Improvement \\
\hline $\begin{array}{c}\text { Convergent } \\
\text { Share Changes }\end{array}$ & $|X|>W>0$ & $W<0$ \\
\hline $\begin{array}{c}\text { Divergent } \\
\text { Share Changes }\end{array}$ & $W>|X| \geq 0$ & Impossible \\
\hline
\end{tabular}

To reiterate in intuitive terms the results derived so far: When inequality falls, both the transfers involved for the anonymous equalization and the positional re-ranking are convergent (i.e. from richer to poorer). Yet when inequality rises, there will be disequalizing transfers that change the shape of the income distribution together with equalizing transfers due to the positional swap. Whether there will be convergence or divergence in shares according to equation (3) will depend on which force dominates.

\footnotetext{
${ }^{6}$ The proof of the other cells in Table 3 is very similar to the one of Proposition 2.

${ }^{7}$ As is well known, when Lorenz curves cross, a Lorenz-consistent measure can always be found showing rising inequality and another Lorenz-consistent measure can be found showing falling inequality.
} 


\subsubsection{Lorenz Dominance, Absolute Lorenz Dominance, and Changes in Dollars}

While the previous section establishes a clear connection between change in inequality as gauged by the Lorenz criterion and share changes, on many occasions our interest is not the changes in shares but the changes in dollars. In particular, often when someone is interested in finding out whether "the rich got richer and the poor, poorer" the reference is to changes in dollars and not merely in shares.

In this section we establish a condition relating changes in inequality under Lorenz-dominance and a dollar-change regression

$$
\Delta d=\gamma_{d}+\delta_{d} d_{0}+u_{d}
$$

In order to derive such a connection, it is useful to express the dollarchange regression (5) in its final-on-initial form (6)

$$
d_{1}=\alpha_{d}+\beta_{d} d_{0}+u_{d}
$$

and to recall that in such a case convergence will occur whenever $\beta_{d}<1$ (or $\left.\delta_{d}<0\right)$. Similarly, we can define a final-on-initial share regression

$$
s_{1}=\alpha_{s}+\beta_{s} s_{0}+u_{s}
$$

Using these regressions we can establish the following result.

Lemma 3. Let $\mu_{t}$ denote the mean income in period $t, \beta_{d}$ and $\beta_{s}$ denote the convergence coefficients given by regressions (6) and (7) in dollars and in shares, respectively, and $g$ denote the economy-wide growth rate in incomes between year 0 and year 1 . Then

$$
\beta_{d}=\beta_{s} \frac{\mu_{1}}{\mu_{0}}=\beta_{s}(1+g) .
$$

Proof: See Appendix.

As before, we can derive a necessary condition relating dollar-changes and Lorenz Dominance, as long as there is negative growth (i.e., $g<0$ ).

Proposition 3. Lorenz Dominance and Convergent/Divergent Dollar Changes Under Negative Growth.

In the case of negative growth $(g<0)$, then: 
i) A Lorenz-improvement $\left(L C_{1} \succ L C_{0}\right)$ implies convergence in dollars $\left(\delta_{d}<0\right)$.

ii) If dollar changes are non-convergent $\left(\delta_{d} \geq 0\right)$ then either a weak Lorenzworsening has taken place $L C_{0} \succeq L C_{1}$, or the Lorenz curves of incomes in periods 0 and 1 cross.

Proof: See Appendix.

Similarly to the previous section, we can derive a series of conditions under which each combination of convergent/divergent dollar-changes can occur along with Lorenz improvement/worsening. These conditions are summarized in Table 4 for each growth scenario. ${ }^{8}$

\section{Table 4: Conditions for Convergent/Divergent Dollar Changes under Lorenz Dominance}

\begin{tabular}{|c|c|c|}
\multicolumn{2}{c}{ A: Economic Growth $(g>0)$} \\
\hline & Lorenz-Worsening & Lorenz-Improvement \\
\hline Convergent & $|X|>W>0$ & $W<0$ \\
Dollar & and & and \\
Changes & $\beta_{s}(1+g)<1$ & $\beta_{s}(1+g)<1$ \\
\hline Divergent & $W>0$ & $W<0$ \\
Dollar & and & and \\
Changes & $1<\beta_{s}(1+g)$ & $1<\beta_{s}(1+g)$ \\
\hline
\end{tabular}

B: Economic Decline $(g<0)$

\begin{tabular}{|c|c|c|}
\hline & Lorenz-Worsening & Lorenz-Improvement \\
\hline Convergent & $W>0$ & \\
Dollar & and & $W<0$ \\
Changes & $\beta_{s}(1+g)<1$ & \\
\hline Divergent & $W>|X| \geq 0$ & \\
Dollar & and & Impossible \\
Changes & $1<\beta_{s}(1+g)$ & \\
\hline
\end{tabular}

For $X$ and $W$ defined in equation (4).

\footnotetext{
${ }^{8}$ The conditions can be easily derived from Lemmas 1-3 and Proposition 2. A proof is available from the authors upon request.
} 
The $(1,1)$ cell in part A states that in periods of economic growth, in order to have convergent dollar changes together with Lorenz-worsening, we need to have income changes large enough such that the convergence brought by the positional changes $(X<0)$ dominates the divergence brought by the widening distribution $(W>0)$.

If during periods of economic decline the dollar losses of the poor are larger than those of the rich, i.e., if there is divergence in dollars, then the income share of the rich will grow and so will inequality. This accounts for the impossibility result in cell $(2,2)$ in part B of Table 4.

What if economic growth is positive and dollar changes are divergent? In that case the dollar gains of the initially poor can be smaller than those of the initially rich, yet the share gains of the initially poor can be higher than the share gains of the initially rich (in which case there would be a fall in relative inequality). An example is $[1,5] \rightarrow[3,8]$.

In more precise terms, since a regression of dollar change on initial dollars is not scale independent, in the case of positive economic growth, falling relative inequality will lead to convergence in dollars only if the convergence in shares $\left(\beta_{s}<1\right)$ is stronger than the diverging impact of proportionally rising incomes $(1+g)$.

Suppose that instead of analyzing relative inequality by comparing income ratios, one was interested in analyzing absolute inequality by looking at dollar differences. In this case we can define an absolute Lorenz curve similar to the one defined in section 3.1 (see for instance, Moyes, 1999). More precisely we can define:

\section{Definition. Vector of Final Dollars in Ascending Order.}

Let $P(\cdot)$ be a permutation operator. Then, define $\mathbf{d}_{\mathbf{c}}=\left(d_{1 c}, \ldots, d_{n c}\right)$ as the final dollar vector when incomes are sorted in ascending order of final income, i.e.

$$
\mathbf{d}_{\mathbf{c}} \equiv P\left(\mathbf{d}_{\mathbf{1}}\right) \quad \text { such that } \quad d_{i c} \leq d_{j c} \forall i \leq j
$$

\section{Definition. Absolute Lorenz Dominance.}

Let $d_{j 0}$ be the initial income in dollars of the individual in position $\mathrm{j}$, when incomes are sorted in ascending order of initial income. Let $d_{j c}$ be the final income in dollars of the individual in position $\mathrm{j}$, when incomes are sorted in ascending order of final income. Furthermore, express these incomes in deviations from their respective means, i.e. $\tilde{d}_{j 0}=d_{j 0}-\mu_{0}$ and $\tilde{d}_{j c}=d_{j c}-\mu_{1}$. The final income distribution absolutely Lorenz-dominates the initial one 
whenever

$$
\begin{aligned}
& \tilde{d}_{1 c}+\tilde{d}_{2 c}+\ldots+\tilde{d}_{j c} \geq \tilde{d}_{10}+\tilde{d}_{20}+\ldots+\tilde{d}_{j 0} \text { for } \mathrm{j}=1,2, \ldots, \mathrm{n}-1 \text { and } \\
& \tilde{d}_{1 c}+\tilde{d}_{2 c}+\ldots+\tilde{d}_{j c}>\tilde{d}_{10}+\tilde{d}_{20}+\ldots+\tilde{d}_{j 0} \text { for some } \mathrm{j}<\mathrm{n} .
\end{aligned}
$$

Absolute Lorenz curves will be denoted by $A L C_{t}$, and absolute Lorenz dominance of incomes in period 0 by those in period 1 will be denoted by $A L C_{1} \succ A L C_{0}$.

In spite of not having a relation of necessity between Lorenz-improvements and convergent dollar changes under all growth scenarios, we can establish such a relation between absolute Lorenz-improvements and convergence in dollars. More specifically, when absolute inequality falls, as judged by the absolute Lorenz-criterion, then it must be the case that the changes in dollars are convergent. This result is stated next.

Proposition 4. Absolute Lorenz Dominance and Convergent/Divergent Dollar Changes

i) An absolute Lorenz-improvement $\left(A L C_{1} \succ A L C_{0}\right)$ implies dollar convergence $\left(\delta_{d}<0\right)$.

ii) If dollar changes are non-convergent $\left(\delta_{d} \geq 0\right)$ then either a weak absolute Lorenz-worsening has taken place $A L C_{0} \succeq A L C_{1}$, or the absolute Lorenz curves of incomes in periods 0 and 1 cross.

Proof: See Appendix.

In this paper we will make no further use of absolute Lorenz Dominance, and instead focus solely on relative inequality comparisons.

\subsubsection{Lorenz Dominance and Proportional Income Changes}

In many applications economists have been interested in studying whether proportional income changes are convergent or divergent. In particular they have studied whether on average initially rich individuals had proportional income changes larger than those of initially poor individuals.

Convergence in dollars and divergence in proportional changes cannot coexist in periods of economic growth, since if the initially poor gain more in dollars than the initially rich (i.e., there is convergence in dollars) then proportional changes are necessarily convergent as well. However, the same 
is not true in periods of economic decline. To appreciate this, consider a hypothetical two-person economy with the following income transition

$$
[2,50] \rightarrow[1,45]
$$

where the poor individual lost 1 dollar while the rich one lost 5 dollars. By our definition there is convergence in dollars. Yet the 1-dollar loss represented half of the poor individual's income, while the 5-dollar loss represented only a $10 \%$ loss for the rich individual. Hence, in this example there was convergence in dollars but divergence in proportional changes.

In this section we explore the relationship between proportional changes in income and Lorenz-improvement/worsening.

\section{Log-Income Approximation}

The most common way to measure proportional convergence is by approximating proportional changes by changes in log-income and estimating a double-log regression

$$
\Delta \ln d=\gamma_{\ln }+\delta_{\ln } \ln d_{0}+u_{\ln }
$$

or its equivalent final-on-initial form $\ln d_{1}=\alpha_{\ln }+\beta_{\ln } \ln d_{0}+u_{\ln }$. Similarly a common way of determining whether inequality is increasing or decreasing is to look at the variance of log-incomes.

As we now show, doing things in these ways can be seriously misleading.

Consider the following example:

$$
[1,1,1,1,1,1,1,1,6,9] \rightarrow[1,1,1,1,1,1,1,1,7,8]
$$

The richest person (call him Bill Gates) has transferred $\$ 1$ to the next richest person (call him Carlos Slim), which is a clear Lorenz-improvement. Inequality therefore falls by the Lorenz criterion and accordingly for any Lorenz-consistent inequality measure. However, the variance of log-incomes is not Lorenz-consistent (Foster and Ok, 1999; Cowell, 2011), and it shows an increase from 0.716 to 0.721 despite the Lorenz-improvement. Moreover, by the transfer principle, a rank-preserving transfer of income from the richest person to anyone lower down in the income distribution should be deemed convergent, and yet if we regress final log-dollars on initial log-dollars, we 
obtain $\beta_{\ln }=1.00045>1$, and hence find divergence in log-dollars. Thus, in this example, a Lorenz-improvement has taken place and yet the regression of final log-income on initial log-income registers divergence and the variance of log-incomes increases (which it must by the Furceri, Wodon-Yitzhaki theorem). The reader is hereby forewarned to be cautious about using logincomes and their variances.

As shown in Table 2, we can find all possible combinations of falling/rising inequality with convergent/divergent log-income changes. In particular, contrary to the share-change case, we can find examples that make compatible falling inequality as gauged by a Lorenz-improvement and divergent logincome changes.

The previous examples illustrate a more general point: that log-incomes can be divergent if a progressive transfer occurs sufficiently high up in the income distribution.

More precisely, we can show the following lemma for a single rank-preserving transfer that is sufficiently small:

Lemma 4. A Single Rank-Preserving Transfer and Convergence/ Divergence of Log-income Changes.

Let gm denote the geometric mean of income at period 0 , and $\exp (1)=2.718$. Consider two individuals $i$ and $j$ such that $d_{0 i}>d_{0 j}>g m * \exp (1)$. Let $h>0$ be a sufficiently small rank-preserving transfer between $i$ and $j$.

a) If such a transfer $h$ is equalizing, it produces a Lorenz-improvement $L C_{1} \succ$ $L C_{0}$, rising inequality as gauged by the log-variance $\left(V\left(\ln d_{1}\right)>V\left(\ln d_{0}\right)\right)$, and a divergent regression coefficient $\left(\delta_{\ln }>0\right)$.

b) If such a transfer $h$ is disequalizing, it produces a Lorenz-worsening $L C_{1} \prec$ $L C_{0}$, falling inequality as gauged by the log-variance $\left(V\left(\ln d_{1}\right)<V\left(\ln d_{0}\right)\right)$, and a convergent regression coefficient $\left(\delta_{\ln }<0\right)$.

Proof: See Appendix.

Lemma 4 suggests why it would be easy to misinterpret a log-change regression like (9). The log-change regression can indicate divergence, as we define it, even when the income changes lead to a Lorenz-improvement. Rank-preserving equalizations which occur sufficiently high up in the income distribution can lead to divergence in log-dollars. This is an unappealing property of log-income regressions such as (9). ${ }^{9}$

\footnotetext{
${ }^{9}$ It can be shown that if incomes follow a log-normal or a Pareto distribution then such misinterpretations by (9) cannot arise. A proof is available from the authors upon request.
} 


\section{True Proportional Changes}

One alternative to the log-income changes regression (9) is to regress the true proportional change in incomes on initial income, namely

$$
\operatorname{pch} \equiv\left(d_{1}-d_{0}\right) / d_{0}=\phi+\theta d_{0}+e .
$$

In this case, if $\theta$ is positive we will say there is divergence in proportions, if $\theta$ is negative we will say there is convergence in proportions, and if $\theta$ equals zero we will say the proportional changes are equal with respect to initial income.

In the case of regression (10) we can show the following results linking inequality changes and true proportional changes.

\section{Proposition 5. Lorenz Dominance and Convergence/Divergence of True Proportional Changes.}

i) A Lorenz-improvement $\left(L C_{1} \succ L C_{0}\right)$ implies convergence in true proportional changes $(\theta<0)$.

ii) If the true proportional changes are non-convergent $(\theta \geq 0)$ then either a weak Lorenz-worsening has taken place $\left(L C_{0} \succeq L C_{1}\right)$, or the Lorenz curves of incomes in periods 0 and 1 cross.

Proof: See Appendix.

Furthermore, we can establish a precise condition for when there will be a Lorenz-worsening despite the existence of convergent proportional changes.

Corollary 1. Lorenz-Worsening and Convergent Proportional Changes. Let $\mathbf{s}_{\mathbf{c}}$ be defined as in (1). Assume there is Lorenz-worsening $\left(L C_{0} \succeq\right.$ $\left.L C_{1}\right)$. If

$$
\frac{1}{n} \sum_{i} \frac{s_{i 1}-s_{i c}}{s_{i 0}}>\left|\frac{1}{n} \sum_{i} \frac{s_{i c}-s_{i 0}}{s_{i 0}}\right|,
$$

then proportional changes will be convergent.

Proof: See Appendix.

The intuition is the same as before: if income changes are large enough, and in a suitable pattern, we can have positional changes, rising inequality, and convergent proportional changes all taking place at the same time. 


\subsection{Relative Inequality Measures and Income Changes}

As mentioned in section 2 researchers often turn to specific inequality measures instead of just comparing Lorenz curves across distributions because of the incompleteness and lack of cardinality of the Lorenz-criterion. For that reason, in this section we present results pertaining to specific commonly used inequality measures.

\subsubsection{Lorenz-Consistent Inequality Measures and Income Changes}

Researchers often use Lorenz-consistent inequality measures like the Gini, Theil, and other indices because whenever the Lorenz-criterion provides an ordering of the inequalities of two distributions, they agree with such ordering.

In section 3.2 we derived a set of results relating income changes with Lorenz Dominance, hence all those results also apply to Lorenz-consistent inequality indices whenever there are no crossings of Lorenz curves. In other words, the results in section 3.2 implicitly apply as well to this family of indices whenever the Lorenz-criterion provides an ordering between distributions. ${ }^{10}$

The study of the relationship between income changes and changes in inequality that involve crossing Lorenz-curves is deferred until section 3.4.

In addition to the previous results, we also derive a relation between the Coefficient of Variation and income changes measured in dollars.

Proposition 6. Convergent/Divergent Dollar Changes, Changing Coefficient of Variation, and Economic Growth.

Let $\beta_{d}$ be defined by the final-on-initial dollar regression (6), and denote the correlation coefficient from this regression by $r_{l}$. Let $C V\left(\mathbf{d}_{\mathbf{t}}\right)$ denote the coefficient of variation of income at period $t$, and let $g$ denote the economywide growth rate in incomes between year 0 and year 1 . Then there is divergence/convergence in dollars (i.e. $\beta_{d} \gtrless 1$ or $\delta_{d} \gtrless 0$ ) as:

$$
r_{l} \frac{C V\left(\mathbf{d}_{\mathbf{1}}\right)}{C V\left(\mathbf{d}_{\mathbf{0}}\right)}(1+g) \gtrless 1 .
$$

\footnotetext{
${ }^{10}$ Wodon and Yitzhaki (2006) derive a relation between changes in the Gini and a convergence coefficient in a so-called "Gini-regression" of final on initial income. The structure of their result is similar to the one of Proposition 3, in that under negative growth a falling Gini index implies convergence in the Gini-regression coefficient, and divergence in this coefficient implies a rising Gini index.
} 
Proof: See Appendix.

Equation (11) shows that to make rising inequality as measured by the coefficient of variation compatible with convergent dollar changes we must either have economic decline $(g<0)$ or a low $r_{l}$. In particular, under economic growth $(g>0)$ and rising coefficients of variation, $r_{l}$ must be small or negative in order to have convergence. In practice, empirical studies find $0<r_{l}<1$. These properties give us the following corollary to Proposition 6 .

\section{Corollary 2. Imperfect Fit, Rising Inequality, and Convergent Dollar Changes With Positive Growth.}

In order for rising inequality as measured by the coefficient of variation and convergent dollars changes to coexist in times of positive growth, the correlation between final income in dollars $\mathbf{d}_{\mathbf{1}}$ and initial income in dollars $\mathbf{d}_{\mathbf{0}}$ must be a sufficiently small positive number or negative. In particular, rising inequality and convergence in dollars can coexist as long as

$$
r_{l}<\frac{C V\left(\mathbf{d}_{\mathbf{0}}\right)}{(1+g) C V\left(\mathbf{d}_{\mathbf{1}}\right)}<1 .
$$

The condition of a low $r_{l}$ reflects what we knew from before, namely that convergence and rising inequality occur when there are large income changes, such that people switch positions as their incomes change from one period to the other.

Equation (11) also serves to illustrate what we already have seen in Tables 2 and 4 , namely that it is possible for divergent dollar changes $\left(\beta_{d}>1\right)$ and falling relative inequality (i.e. $C V\left(\mathbf{d}_{\mathbf{0}}\right)>C V\left(\mathbf{d}_{\mathbf{1}}\right)$ ) to coexist when income growth $g$ is strong enough, due to the scale-dependence of dollar change regressions.

\subsubsection{Lorenz Inconsistent Inequality Measures and Income Changes: The Case of the Variance.}

In the macro and labor literatures, it is common to assess changes in relative inequality by focusing on the variance of log-incomes. In spite of its Lorenzinconsistency, the variance of logs remains quite popular in the literature. In this section we present the basic relationship between changes in inequality as measured by the variance of logs and the coefficient in a log-change regression (9). This result was derived independently by Furceri (2005) and Wodon and Yitzhaki (2006), and we present it next. 
Lemma 5. Log-Income Convergence and Variance of Log-Income. If $\Delta V(\ln d)<0$, then $\delta_{\ln }<0$, where $\delta_{\ln }$ is defined by the regression coefficient in (9).

Rather than presenting a proof of this result, we include a slightly more general result linking the variance of any monotonically increasing function of income $y=f(d)$ (e.g. logarithms, shares, dollars, etc.) and the coefficient of a regression of the changes in this generic variable $y$ on its initial level $y_{0}$. Namely, we present a result concerning the relationship between the changes in $V(y)=V(f(d))$ and the coefficient $\delta_{y}$ in a regression of $\Delta y$ on $y_{0}$

$$
\Delta y=\gamma_{y}+\delta_{y} y_{0}+u_{y}
$$

Proposition 7. Linear Convergence and Changes in Variance for the Class of Monotonic Transformations of Income in Dollars. Let $f(d)$ be any monotonically increasing function of income in dollars and denote the value of this function by $y$. Consider the change regression given by (12). Then:

i) If $\Delta V(y)<0$, then $\delta_{y}<0$.

ii) If $\delta_{y} \geq 0$ then $\Delta V(y) \geq 0$.

Proof: See Appendix.

Proposition 7 shows that divergence in the changes of a monotonically increasing function of income $y=f(d)$ implies a rising variance of this function, (or alternatively a falling variance of $y$ implies convergent changes of $y$ ). However, as before convergence does not imply a falling variance: $\delta_{y}<0 \nRightarrow \Delta V(y)<0$.

We can also establish a precise condition for when we can observe rising inequality, as measured by the variance of $y$, and convergent changes in $y$.

Corollary 3. Convergent Changes, Rising Variance, and Variance of Changes.

If

$$
0<\Delta V(y)<V(\Delta y)
$$

then $\delta_{y}<0$.

Proof: See Appendix.

To reemphasize, these results pertain to any monotonically increasing function of income, as long as we use the same function $y=f(d)$ as dependent and independent variables, i.e. as long as we run share-changes on initial shares, log-income changes on initial log-incomes, etc. 


\subsection{Extensions to Cases of a Single-Lorenz Crossing from above}

So far, many of our results have been derived by analyzing rising or falling inequality as judged by Lorenz-worsenings or improvements. Theoretically, it is possible for the Lorenz curves of two distributions to cross, which often happens in practice.

As some of the previous counter-examples have shown, the extension of our results to cases of Lorenz-crossings is not straightforward, since several ambiguities could arise. However, we can still find a relationship between convergence coefficients $\delta$ and certain inequality measures under a particular type of Lorenz-crossing. In particular, we will focus on a single crossing from above as defined next.

\section{Definition. Single Lorenz Crossing From Above.}

Denote by $L C(\mathbf{d} ; p)$ the Lorenz curve ordinate corresponding to the lowest $100 \mathrm{p} \%$ of income recipients, for $p \in[0,1]$. The Lorenz curve for a distribution $\mathbf{d}$ is said to intersect that of $\mathbf{d}^{\prime}$ once from above iff there exists $p^{*} \in(0,1)$ and intervals $P \equiv\left[0, p^{*}\right]$ and $P^{\prime} \equiv\left[p^{*}, 1\right]$ such that

$$
\begin{aligned}
& L C(\mathbf{d} ; p) \geq L C\left(\mathbf{d}^{\prime} ; p\right) \quad \forall p \in P \quad \text { and }>\text { for some } p \in P \\
& L C(\mathbf{d} ; p) \leq L C\left(\mathbf{d}^{\prime} ; p\right) \quad \forall p \in P^{\prime} \quad \text { and }<\text { for some } p \in P^{\prime} \text {. }
\end{aligned}
$$

To better understand the welfare properties of the inequality assessments under this type of crossing we need to define the property of "transfer sensitivity" of an inequality measure.

Definition. Transfer-Sensitive Inequality Measures (Shorrocks and Foster, 1987)

An inequality measure $I()$ is transfer sensitive (TS) iff $I\left(\mathbf{d}_{\mathbf{0}}\right)>I\left(\mathbf{d}_{\mathbf{1}}\right)$ whenever $\mathbf{d}_{\mathbf{1}}$ is obtained from $\mathbf{d}_{\mathbf{0}}$ by a series of transfers whereby at each stage i) a progressive transfer occurs at lower income levels, ii) a regressive transfer occurs at higher income levels, iii) ranks remain unchanged, and iv) the variance of incomes remains unchanged.

Intuitively speaking, a transfer-sensitive inequality measure is one that records a fall in inequality whenever there is a progressive transfer at the lower part of the income distribution in tandem with a regressive transfer at 
higher income levels, to the extent that the transfers are comparable in the sense required by condition iv) in the above definition. ${ }^{11}$

In other words, transfer-sensitive inequality measures can rank certain pairs of distributions in the presence of Lorenz-crossings by giving greater weight to transfers that occur in the lower part of the income distribution. Shorrocks and Foster (1987) show that the Atkinson family and the Generalized Entropy class with parameter less than two satisfy the transfer-sensitive property, but the Gini coefficient does not.

An important result due to Shorrocks and Foster (1987) provides a relation between transfer-sensitive inequality indices and the Coefficient of Variation, CV. For the sake of completeness we reproduce it next without proof.

Proposition 8. Single Lorenz Crossing from Above, Transfer Sensitive Inequality Indices and the Coefficient of Variation. (Shorrocks and Foster, 1987)

If the Lorenz curve of $\mathbf{d}_{\mathbf{1}}$ intersects that of $\mathbf{d}_{\mathbf{0}}$ once from above, then:

a) $I\left(\mathbf{d}_{\mathbf{0}}\right)>I\left(\mathbf{d}_{\mathbf{1}}\right)$ for all inequality measures $I_{T S}()$ satisfying transfer sensitivity $(\boldsymbol{T} \boldsymbol{S})$, scale-independence $(\boldsymbol{S})$, and population-independence $(\boldsymbol{P})$

if and only if

b) $C V\left(\mathbf{d}_{\mathbf{0}}\right) \geq C V\left(\mathbf{d}_{\mathbf{1}}\right)$.

Since the $C V^{2}$ is the variance of shares, ${ }^{12}$ we can use Propositions 7 and 8 to establish a result linking the coefficient in a regression of changes in shares on initial share (i.e. $\delta_{s}$ ), to the changes in Transfer Sensitive inequality indices, $I_{T S}$, when there is a single-crossing from above in Lorenz curves.

Proposition 9. Single Lorenz-Crossing from Above, Transfer Sensitive Inequality Indices, and Convergent Share Changes

Let the Lorenz curve of $\mathbf{d}_{\mathbf{1}}$ intersect that of $\mathbf{d}_{\mathbf{0}}$ once from above. For all $I_{T S}()$ as defined in Proposition 8, if $C V\left(\mathbf{d}_{\mathbf{0}}\right) \geq C V\left(\mathbf{d}_{\mathbf{1}}\right)$, then both $\delta_{s} \leq 0$ and $I_{T S}\left(\mathbf{d}_{\mathbf{0}}\right)>I_{T S}\left(\mathbf{d}_{\mathbf{1}}\right)$.

Proof: See Appendix.

This result is weaker than Proposition 2.i), because it does not say that a single crossing from above will imply share-convergence. Instead it states

\footnotetext{
${ }^{11} \mathrm{~A}$ formal statement together with a careful discussion of the concept is presented in Shorrocks and Foster (1987).

${ }^{12}$ This is shown in the proof of Proposition 9.
} 
that if the Lorenz curve of final-period incomes crosses that of initial incomes once from above and the Coefficient of Variation falls, then there will be both share-convergence and a fall in inequality according to all relative inequality measures that satisfy transfer sensitivity (TS), scale independence (S), and population independence $(\mathbf{P})$.

To illustrate empirically Proposition 9 consider the transition

$$
\mathbf{d}_{\mathbf{0}}=[1,5,10,11] \rightarrow \mathbf{d}_{\mathbf{1}}=[2,4,9,12] \text {. }
$$

In this case the conditions of the Proposition are satisfied, namely there is: i) a single-crossing from above in the Lorenz curves, and ii) a falling $\mathrm{CV}$. In this case it is readily verified that commonly used indices like the Atkinson family and Generalized Entropy with parameter $<2$ will mark a reduction in inequality, and there is share convergence as well. In this case, the Atkinson index with parameter 0.5 falls from 0.126 to 0.096 and the Generalized Entropy index with parameter 1 falls from 0.218 to 0.184 . There is share convergence as well: in this situation, $\delta_{s}=-0.046$.

To appreciate the importance of having a falling Coefficient of Variation in Proposition 9, consider the example $\mathbf{d}_{\mathbf{0}}=[1,5,10] \rightarrow \mathbf{d}_{\mathbf{1}}=[2,4,25]$. In this case the Lorenz curve of $\mathbf{d}_{\mathbf{1}}$ crosses that of $\mathbf{d}_{\mathbf{0}}$ once from above, yet the Coefficient of Variation does not fall, and we cannot appeal to Proposition 9 .

Finally, notice that Proposition 9 and Lemma 3 could be combined to derive conditions relating changes in Transfer-Sensitive inequality measures to income changes in dollars.

This concludes our derivation of results. We turn now to a summary of the results and a concluding discussion.

\section{Summary of Results and Concluding Ob- servations}

This paper has explored mathematically the relationship between changing income inequality in the cross section and panel income changes. All four combinations rising inequality and convergent panel income changes, rising inequality and divergent panel income changes, falling inequality and convergent panel income changes, and falling inequality and divergent panel income changes - have been shown possible (Table 2). Conditions for the 
various possibilities have been derived in Section 3. The sources of results for each combination of rising or falling income inequality, convergent or divergent panel income changes, and positive or negative economic growth, are summarized for convenience in Table 5. Each cell of the table refers to the proposition, corollary, or lemma where the result is derived.

Three observations about the results are particularly trenchant.

First, the great majority of results are derived measuring inequality change by Lorenz-improvements and Lorenz-worsenings, which are particularly powerful criteria for making inequality comparisons. Thus, all who agree on the desirability of using Lorenz criteria would feel confident that the various combinations involve "good" ways of measuring inequality.

Second, some of the results require Lorenz crossings or hold for a carefully chosen inequality index but not for all Lorenz-consistent indices. Consequently, these results are weaker than those based on Lorenz-dominance.

And third, some combinations are impossible, but there are only a few of them. One impossibility is the one previously proved by Furceri (2005) and Wodon and Yitzhaki (2006), namely that it is impossible to have divergent log-dollar changes and falling relative inequality as measured by the variance of logs. However, it is possible to have divergent log-dollar changes and a Lorenz-improvement, hence falling inequality as measured by any Lorenzconsistent index. The prior impossibility result is due to the authors' use of an inequality index which is not Lorenz-consistent. The other impossibilities are ones which we have proven here and were not previously in the literature (Propositions 2 - 5 and Tables 3-4). One is that we cannot simultaneously have divergent panel income changes in shares and a Lorenz-improvement, either in times of economic growth or in times of economic decline. Another is that in times of economic decline we cannot simultaneously have divergent panel income changes in dollars and a Lorenz-improvement. Furthermore, we cannot simultaneously have divergent dollar changes and an absolute Lorenzimprovement, either in times of growth or decline. Finally, we cannot have divergent true proportional changes and a Lorenz-improvement, either in times of economic growth or in times of economic decline.

Every other combination of rising or falling income inequality, divergent or convergent panel income changes, and economic growth or decline is possible, and we have displayed the conditions under which each arises. 
Table 5: Roadmap of Results

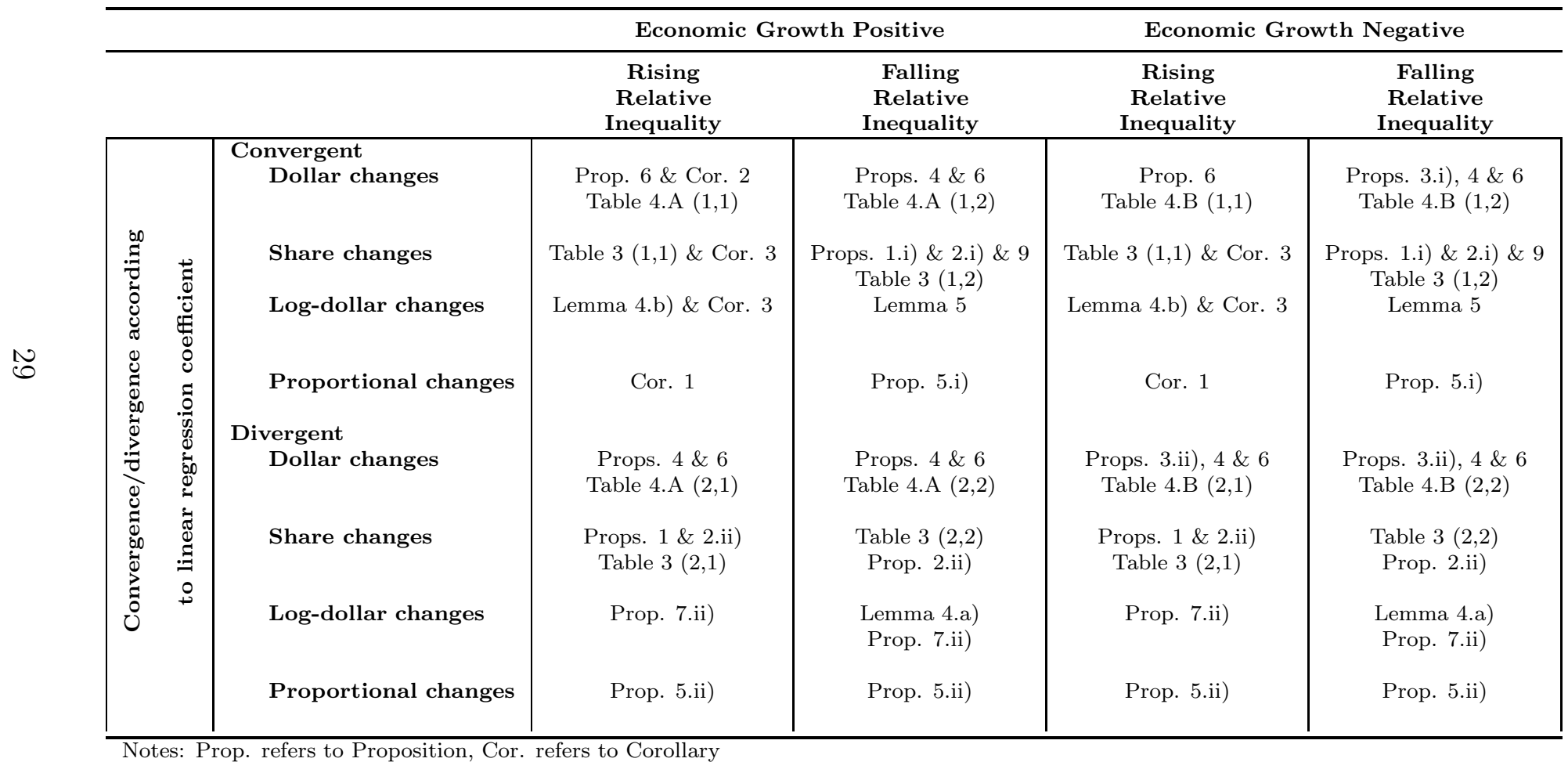


To conclude let us return to where we started; namely with the reconciliation between i) convergent panel income changes and rising inequality, and between ii) divergent panel income changes and falling inequality.

The reason that convergence can occur in spite of rising inequality, is that panel income changes can be large enough such that some initially lowearners become high earners in a widening distribution. In fact, it is precisely because panel studies abandon the property of cross-sectional anonymity that such pattern can be identified.

In addition, the coexistence of divergent panel income changes and falling inequality depends crucially on the way inequality and divergence are measured. In particular, we show that dollar changes can be divergent in conjunction with Lorenz-improvements if income growth is positive and large enough. The reason for this is that a regression of dollar change on initial dollars is not scale independent; while relative inequality measures are. However, the impossibility of simultaneous having divergent dollar changes and falling inequality reappears if we focus on absolute inequality and if there is an absolute Lorenz-improvement. Researchers who consider a desirable property to have convergence whenever there is a narrowing of the dispersion of the income distribution, should then be careful when choosing the inequality measures and income change regressions they pair together.

The other instance where we can have divergence together with Lorenzimprovements occurs for regressions in log-dollars. More specifically, we showed that equalizing transfers that occur sufficiently high up in the income distribution will lead to a reduction in inequality by any measure that satisfies the transfer principle, yet the log-dollar regression will register divergence.

The results derived in this paper open up additional questions as to the nature of individual income changes. For instance, when rising inequality is observed together with convergent panel income changes, is this finding driven by a few individuals experiencing large changes, or by many individuals experiencing moderate changes, or are both important? Exploring the precise way in which these large individual changes occur is an important question for future research. 


\section{References}

Atkinson, A. B. and F. Bourguignon (eds.) (2000), Handbook of Income Distribution, vol. 1, Amsterdam: Elsevier.

Atkinson, A. B. and F. Bourguignon (eds.) (2015), Handbook of Income Distribution, vol. 2, Amsterdam: Elsevier.

Barro, R. J. (1991), "Economic Growth in a Cross Section of Countries", Quarterly Journal of Economics, vol. 106(2): 407-443.

Bliss, C. (1999), "Galton's Fallacy and Economic Convergence", Oxford Economic Papers, vol. 51(1): 4-14.

Cowell, F. A. (2011), Measuring Inequality, Oxford: Oxford University Press, 3rd edn.

Durlauf, S. N. and D. Quah (1999), "The New Empirics of Economic Growth", in Handbook of Macroeconomics, (eds.) J. Taylor and M. Woodford, Elsevier, vol. 1, pp. 235-308.

Fields, G. S. (2008), "Income Mobility", in The New Palgrave Dictionary of Economics, (eds.) L. Blume and S. Durlauf, Palgrave Macmillan.

Fields, G. S. (2010), "What We Know (and Want to Know) about Earnings Mobility in Developing Countries", In World Bank "Earnings Mobility Analysis".

Fields, G. S. and J. C. Fei (1978), "On Inequality Comparisons", Econometrica, vol. 46(2): 303-316.

Foster, J. E. and E. A. Ok (1999), "Lorenz Dominance and the Variance of Logarithms", Econometrica, vol. 67(4): 901-907.

Furceri, D. (2005), " $\beta$ and $\sigma$-convergence: A mathematical relation of causality", Economics Letters, vol. 89: 212-215.

Jäntti, M. and S. P. Jenkins (2015), "Income Mobility", in Handbook of Income Distribution, (eds.) A. Atkinson and F. Bourguignon, Elsevier, vol. 2, pp. 807-935. 
Korotayev, A., J. Zinkina, J. Bogevolnov, and A. Malkov (2011), "Global Unconditional Convergence among Larger Economies after 1998?", Journal of Globalization Studies, vol. 2(2): 25-62.

Moyes, P. (1999), "Stochastic Dominance and the Lorenz Curve", in Handbook of Income Inequality Measurement, (ed.) J. Silber, Springer Netherlands, vol. 71 of Recent Economic Thought Series, pp. 199-225.

Sala-i-Martin, X. (1996), "The Classical Approach to Convergence Analysis", Economic Journal, vol. 106: 1019-1036.

Sen, A. (1997), On Economic Inequality, Oxford: Oxford University Press, expanded edition with a substantial annexe by James E. Foster and Amartya Sen.

Shorrocks, A. and J. E. Foster (1987), "Transfer Sensitive Inequality Measures", The Review of Economic Studies, vol. LIV: 487-497.

Solon, G. (1999), "Intergenerational Mobility in the Labor Market", in Handbook of Labor Economics, (eds.) O. Ashenfelter and D. Card, Amsterdam: Elsevier, vol. 3, chap. 29, pp. 1761-1800.

Wodon, Q. and S. Yitzhaki (2006), "Convergence forward and backward?", Economics Letters, vol. 92: 47-51. 


\section{Appendix}

\section{Proofs of Propositions}

\section{Lemma 1. Proof of part ii)}

Lorenz Worsening implies $W>0$.

Let $\mathbf{s}_{\mathbf{0}}$ be the initial vector of shares and $\mathbf{s}_{\mathbf{c}}$ be defined in (1).

Theorem 2.1 in Fields and Fei (1978) implies that if the distribution of $\mathbf{s}_{\mathbf{0}}$ Lorenz-dominates that of $\mathbf{s}_{\mathbf{c}}$, i.e. if $L C_{0} \succ L C_{c}$, then it is possible to go from $\mathbf{s}_{\mathbf{0}}$ to $\mathbf{s}_{\mathbf{c}}$ by means of a series of rank-preserving disequalizing transfers.

One convenient way of representing such transfers is by indexing them as $h_{i j}$ where the first index, i, indicates who is making a transfer and the second one, $\mathrm{j}$, who is receiving it.

Since the transfers are disequalizing, and no one makes a transfer to himself, they satisfy the conditions:

$$
\begin{array}{ll}
h_{i j}=0 & \text { for } i \geq j \\
h_{i j} \geq 0 & \text { for } i<j \quad \text { with strict inequality for some }\{i, j\} .
\end{array}
$$

The total transfers made by individual i will be the sum over the second index j, namely

$$
h_{i .}=\sum_{j=1}^{n} h_{i j} .
$$

Similarly, the total transfers received by this same individual will be the sum over the first index, namely

$$
h_{\cdot i}=\sum_{j=1}^{n} h_{j i} .
$$

Hence, the change in this person's income share can be expressed as the difference in the two previous quantities, i.e.

$$
s_{i c}-s_{i 0}=h_{\cdot i}-h_{i}=\sum_{j=1}^{n} h_{j i}-\sum_{j=1}^{n} h_{i j} .
$$

By construction, the sum of the share changes over all individuals is zero, hence each person's share loss is somebody else's share gain, and also each 
share gain is somebody else's loss. In other words, the transfers $h_{i j}$ appear with a positive sign in the share change of individual $\mathrm{j}$, and with a negative sign in the share change of individual i. Furthermore, the sender i is always poorer than the receiver $\mathrm{j}$, since the transfer is disequalizing. Hence, for each transfer $h_{i j}$ we have

$$
h_{i j} s_{j 0}-h_{i j} s_{i 0}=h_{i j}\left(s_{j 0}-s_{i 0}\right) \geq 0 .
$$

Hence, $W$ can be rewritten as

$$
\begin{aligned}
W & =n^{-1} \sum_{i}\left(s_{i c}-s_{i 0}\right) s_{i 0} \\
& =n^{-1} \sum_{i}\left(\sum_{j=1}^{n} h_{j i}-\sum_{j=1}^{n} h_{i j}\right) s_{i 0} .
\end{aligned}
$$

That is, $W$ will be the average of terms $h_{i j}\left(s_{j 0}-s_{i 0}\right)$ for all the transfers $h_{i j}$. Since these terms are non-negative, and some will be strictly positive, then $W$ will be positive.

In other words, we have shown that $L C_{0} \succ L C_{c}$ implies $W>0$. However, by construction, the Lorenz curve of the vector $\mathbf{s}_{\mathbf{c}}$ is the same as that of the final income vector $\mathbf{s}_{\mathbf{1}}$ (i.e. $L C_{c}=L C_{1}$ ), so we have that $L C_{0} \succ L C_{1}$ implies $W>0$.

The proof of part i) follows by reproducing the previous steps, now with rank-preserving equalizing transfers.

Lemma 2. Recall $\mathbf{s}_{\mathbf{c}}$ is a permutation of $\mathbf{s}_{\mathbf{1}}$. Since both vectors have the same distribution, the only changes are the ones due to positional swaps. If nobody changes positions $\mathbf{s}_{\mathbf{c}}=\mathbf{s}_{\mathbf{1}}$, and $X=0$, trivially.

Otherwise, any positional swap will imply the transfer of resources $g_{k l}$ from individual $\mathrm{k}$ to individual $\mathrm{l}$ where $\mathrm{k}$ is initially richer than 1 , i.e. $l<k$. Moving from $\mathbf{s}_{\mathbf{c}}$ to $\mathbf{s}_{\mathbf{1}}$ will imply a series of such positional swaps.

The total transfers made by individual $\mathrm{i}$ when going from $\mathbf{s}_{\mathbf{c}}$ to $\mathbf{s}_{\mathbf{1}}$ will be the sum

$$
g_{i .}=\sum_{j=1}^{n} g_{i j}
$$


while the total transfers received by this same individual during this transition will be the sum

$$
g_{\cdot i}=\sum_{j=1}^{n} g_{j i} .
$$

The change in this person's income share from such transfers can be then expressed as

$$
s_{i 1}-s_{i c}=g_{\cdot i}-g_{i}=\sum_{j=1}^{n} g_{j i}-\sum_{j=1}^{n} g_{i j} .
$$

The transfers $g_{k l}$ appear once with a positive sign and once with a negative sign each. Furthermore, as we established before, in both cases the sender is always richer than the receiver. Hence, for each transfer $g_{k l}$ we have that the product

$$
g_{k l} s_{l 0}-g_{k l} s_{k 0}=g_{k l}\left(s_{l 0}-s_{k 0}\right)
$$

is negative. Hence, the term

$$
X=n^{-1} \sum_{i}\left(s_{i 1}-s_{i c}\right) s_{i 0}=n^{-1} \sum_{i}\left(\sum_{j=1}^{n} g_{j i}-\sum_{j=1}^{n} g_{i j}\right) s_{i 0}
$$

will be the average of terms $g_{k l}\left(s_{l 0}-s_{k 0}\right)$ for all transfers $g_{k l}$. Since all these terms are non-positive, and some will be strictly negative, then $\mathrm{X}$ will be negative as well.

Proposition 1. Consider the share change regression (3)

$$
\Delta s \equiv s_{1}-s_{0}=\gamma_{s}+\delta_{s} s_{0}+u_{s}
$$

The coefficient $\delta_{s}$ equals

$$
\delta_{s}=\frac{\operatorname{cov}\left(\Delta s, s_{0}\right)}{V\left(s_{0}\right)}
$$

Hence, its sign will be determined by the sign of the covariance

$$
\begin{aligned}
\operatorname{cov}\left(\Delta s, s_{0}\right) & =n^{-1} \sum_{i}\left(s_{i 1}-s_{i 0}\right) s_{i 0}-\overline{\Delta s} \cdot \overline{s_{0}} \\
& =n^{-1} \sum_{i}\left(s_{i 1}-s_{i 0}\right) s_{i 0} \quad \text { (since the average share-change is zero) } \\
& =n^{-1} \sum_{i}\left[\left(s_{i 1}-s_{i c}\right)+\left(s_{i c}-s_{i 0}\right)\right] s_{i 0} \\
& =n^{-1} \sum_{i}\left(s_{i c}-s_{i 0}\right) s_{i 0} \quad \text { (since there are no positional changes). }
\end{aligned}
$$


Hence, the sign of this covariance will equal the sign of $W$, and the result follows from Lemma 1.

Proposition 2. This proposition is easily proven by noting three important facts.

First, as shown in the proof of Proposition 1, the sign of the coefficient $\delta_{s}$ in equation (3) is determined by the sign of the covariance

$$
\begin{aligned}
\operatorname{cov}\left(\Delta s, s_{0}\right) & =n^{-1} \sum_{i}\left(s_{i 1}-s_{i 0}\right) s_{i 0} \\
& =n^{-1} \sum_{i}\left[\left(s_{i 1}-s_{i c}\right)+\left(s_{i c}-s_{i 0}\right)\right] s_{i 0} \\
& =X+W
\end{aligned}
$$

for $X$ and $W$ defined in (4). Hence,

$$
\operatorname{sign}\left(\delta_{s}\right)=\operatorname{sign}(X+W) .
$$

Second, by Lemma $1, L C_{1} \succ L C_{0} \Longrightarrow W<0$.

Finally, by Lemma $2, X \leq 0$.

Hence, if $L C_{1} \succ L C_{0}$ then $X+W<0$, namely $\delta_{s}<0$. Part ii) is just the contrapositive of i).

Lemma 3. The regression in dollars (6) is

$$
d_{1}=\alpha_{d}+\beta_{d} d_{0}+u_{d} .
$$

Dividing this equation by $\mu_{1}$ we obtain

$$
\begin{aligned}
s_{1} & =\frac{\alpha_{d}}{\mu_{1}}+\beta_{d} \frac{d_{0}}{\mu_{1}}+\frac{u_{d}}{\mu_{1}} \\
& =\frac{\alpha_{d}}{\mu_{1}}+\beta_{d} \frac{d_{0}}{\mu_{0}} \frac{\mu_{0}}{\mu_{1}}+\frac{u_{d}}{\mu_{1}} \\
& =\frac{\alpha_{d}}{\mu_{1}}+\beta_{d} s_{0} \frac{\mu_{0}}{\mu_{1}}+u_{s} .
\end{aligned}
$$

Hence,

$$
\alpha_{s}=\frac{\alpha_{d}}{\mu_{1}} ; \beta_{s}=\beta_{d} \frac{\mu_{0}}{\mu_{1}} .
$$

The Lemma follows from this last equation. 
Proposition 3. By Proposition 2 a Lorenz-improvement $\left(L C_{1} \succ L C_{0}\right)$ implies $\delta_{s}<0$, (or $\beta_{s}<1$ ). Also, whenever there is negative growth (i.e., $g<0), 1+g<1$.

Hence, if a Lorenz-improvement occurs in periods with negative growth then $\beta_{d}=\beta_{s}(1+g)<1$.

Part ii) is just the contrapositive of $\mathrm{i}$ ).

Proposition 4. To prove this result it is useful to have first the following definition.

Definition. Translation Improvement.

The income vector $\mathbf{d}_{\mathbf{1}}$ is obtained from $\mathbf{d}_{\mathbf{0}}$ by a translation improvement whenever

$$
\mathbf{d}_{\mathbf{1}}=\mathbf{d}_{\mathbf{0}}+\kappa \mathbf{1},
$$

for some $\kappa>0$, where $\mathbf{1}=(1,1, \ldots, 1)$.

Notice also that the sign of $\delta_{d}$ is determined by the sign of the covariance

$$
\begin{aligned}
\operatorname{cov}\left(\Delta d, d_{0}\right) & =n^{-1} \sum_{i}\left(d_{i 1}-d_{i 0}\right) d_{i 0}-\left(\mu_{1}-\mu_{0}\right) \mu_{0} \\
& =n^{-1} \sum_{i}\left[\left(d_{i 1}-d_{i c}\right)+\left(d_{i c}-d_{i 0}\right)\right] d_{i 0}-\left(\mu_{1}-\mu_{0}\right) \mu_{0} .
\end{aligned}
$$

Proposition 3.3 in Moyes (1999) implies that if the distribution $\mathbf{d}_{\mathbf{c}}$ absolutely Lorenz-dominates that of $\mathbf{d}_{\mathbf{0}}$, i.e. if $A L C_{c} \succ A L C_{0}$, then it is possible to go from $\mathbf{d}_{\mathbf{0}}$ to $\mathbf{d}_{\mathbf{c}}$ by means of a series of rank-preserving equalizing transfers and translation improvements like the one above defined.

As we established in the proof of Lemma 1 the rank-preserving equalizing transfers $h_{i j}$ will be such that:

i) they appear once with a positive sign and once with a negative sign, and

ii) the sender $\mathrm{i}$ is always richer than the receiver $\mathrm{j}$.

Furthermore, the translation improvements will, by construction, addup to the change in means between periods 0 and 1 . More specifically, if $k_{s}, k_{s+1}, \ldots, k_{s+m}$ are a sequence of translation improvements then

$$
\mu_{1}-\mu_{0}=\sum_{l=0}^{m} k_{s+l} .
$$


Hence, the person's i dollar change when going from $d_{i 0}$ to $d_{i c}$ is

$$
\begin{aligned}
d_{i c}-d_{i 0} & =\sum_{j=1}^{n} h_{j i}-\sum_{j=1}^{n} h_{i j}+\mu_{1}-\mu_{0} . \\
& =h_{\cdot i}-h_{i}+\mu_{1}-\mu_{0}
\end{aligned}
$$

This implies that

$$
\begin{aligned}
\operatorname{cov}\left(\Delta d, d_{0}\right) & =n^{-1} \sum_{i}\left[\left(d_{i 1}-d_{i c}\right)+\left(d_{i c}-d_{i 0}\right)\right] d_{i 0}-\left(\mu_{1}-\mu_{0}\right) \mu_{0} \\
& =n^{-1} \sum_{i}\left[\left(d_{i 1}-d_{i c}\right)+\left(h_{\cdot i}-h_{i}+\mu_{1}-\mu_{0}\right)\right] d_{i 0}-\left(\mu_{1}-\mu_{0}\right) \mu_{0} \\
& =n^{-1} \sum_{i}\left[\left(d_{i 1}-d_{i c}\right)+\left(h_{\cdot i}-h_{i}\right)\right] d_{i 0}+\left(\mu_{1}-\mu_{0}\right) \mu_{0}-\left(\mu_{1}-\mu_{0}\right) \mu_{0} \\
& =n^{-1} \sum_{i}\left[\left(d_{i 1}-d_{i c}\right)+\left(h_{\cdot i}-h_{i}\right)\right] d_{i 0}
\end{aligned}
$$

Since the transfers $h_{i j}$ are equalizing, the term

$$
n^{-1} \sum_{i}\left(h_{\cdot i}-h_{i \cdot}\right) d_{i 0}
$$

will be negative, similar to what occurs in the proof of Lemma 1. Furthermore, the term

$$
n^{-1} \sum_{i}\left(d_{i 1}-d_{i c}\right) d_{i 0}
$$

will be non-positive by Lemma 2 .

In summary, we have proven that $A L C_{1} \succ A L C_{0} \Longrightarrow \delta_{d}<0$. Part ii) is just the contrapositive of i).

Lemma 4. Let $\mathrm{gm}$ denote the geometric mean of incomes at period 0, i.e.

$$
g m=\exp \left(n^{-1} \sum_{i} \ln d_{i}\right) .
$$

Let $h>0$ be a sufficiently small rank-preserving transfer. Consider two individuals $\mathrm{i}$ and $\mathrm{j}$ such that $d_{i 0}>d_{j 0}>g m * \exp (1)$ and assume that the only income change when going from period 0 to 1 is the transfer $h$ between $\mathrm{i}$ and $\mathrm{j}$. 
It follows from Fields and Fei (1978) that if the transfer is equalizing it will lead to a Lorenz-improvement, and the opposite will occur if the transfer is disequalizing. The only result to establish is the sign of the coefficient $\delta_{\ln }$ in a log-change regression (9) under the stated conditions.

Consider the case a) of a single rank-preserving equalizing transfer. That is the transfer goes from the richer person i to the poorer person $\mathrm{j}$. Under the stated assumptions the sign of $\delta_{\mathrm{ln}}$ will be determined by the covariance

$$
\operatorname{cov}\left(\Delta \ln d, \ln d_{0}\right)=n^{-1} \sum_{l}\left(\ln d_{l 1}-\ln d_{l 0}\right) \ln d_{l 0}-\overline{\Delta \ln d} \cdot \overline{\ln d_{0}}
$$

Note that all terms in the summation are zero except for $l \in\{i, j\}$, so we have

$$
\begin{aligned}
\operatorname{cov}\left(\Delta \ln d, \ln d_{0}\right) & =n^{-1}\left[\left(\Delta \ln d_{i}\right) \ln d_{i 0}+\left(\Delta \ln d_{j}\right) \ln d_{j 0}\right]-\overline{\Delta \ln d} \cdot \overline{\ln d_{0}} \\
& =n^{-1}\left[\left(\ln \left(d_{i 0}-h\right)-\ln d_{i 0}\right) \ln d_{i 0}\right]+\cdots \\
& \cdots+n^{-1}\left[\left(\ln \left(d_{j 0}+h\right)-\ln d_{j 0}\right) \ln d_{j 0}\right]-\overline{\Delta \ln d} \cdot \overline{\ln d_{0}}
\end{aligned}
$$

A First-order Taylor expansion around $h=0$ for the first two terms is

$$
\begin{aligned}
& n^{-1}\left[\left(\ln \left(d_{i 0}-h\right)-\ln d_{i 0}\right) \ln d_{i 0}\right] \cong-\frac{\ln d_{i 0}}{d_{i 0}} \frac{h}{n} \\
& n^{-1}\left[\left(\ln \left(d_{j 0}+h\right)-\ln d_{j 0}\right) \ln d_{j 0}\right] \cong \frac{\ln d_{j 0}}{d_{j 0}} \frac{h}{n} .
\end{aligned}
$$

A similar expansion for the average log-income change is

$$
\overline{\Delta \ln d} \cong \frac{h}{n}\left(\frac{1}{d_{j 0}}-\frac{1}{d_{i 0}}\right)
$$

Hence, for a marginal transfer $h$

$$
\operatorname{cov}\left(\Delta \ln d, \ln d_{0}\right) \cong \frac{h}{n}\left(\frac{\ln d_{j 0}-\overline{\ln d_{0}}}{d_{j 0}}-\frac{\ln d_{i 0}-\overline{\ln d_{0}}}{d_{i 0}}\right) .
$$

The sign of this covariance will be determined by the behavior of the function

$$
\frac{\ln x-\overline{\ln d_{0}}}{x}
$$

with derivative

$$
\frac{1-\ln x+\overline{\ln d_{0}}}{x^{2}}
$$


This derivative will be negative when

$$
x>\exp (1) * g m .
$$

Hence, if individuals have income $d_{i 0}>d_{j 0}>\exp (1) * g m$,

$$
\frac{\ln d_{j 0}-\overline{\ln d_{0}}}{d_{j 0}}-\frac{\ln d_{i 0}-\overline{\ln d_{0}}}{d_{i 0}}
$$

will have a positive sign and so $\delta_{\ln }>0$. The case of a disequalizing transfer is proved similarly.

Proposition 5. Rewrite the proportional change regression (10) as

$$
\frac{d_{1}}{d_{0}}=(\phi+1)+\theta d_{0}+e
$$

Then the sign of $\theta$ will depend on the sign of the covariance

$$
\begin{aligned}
\operatorname{cov}\left(\frac{d_{1}}{d_{0}}, d_{0}\right) & =E\left(\frac{d_{1}}{d_{0}} d_{0}\right)-E\left(\frac{d_{1}}{d_{0}}\right) \mu_{0} \\
& =\mu_{1}-E\left(\frac{d_{1}}{d_{0}}\right) \mu_{0} .
\end{aligned}
$$

Hence, there will be divergence (i.e. $\theta>0$ ) whenever $\mu_{1}>E\left(\frac{d_{1}}{d_{0}}\right) \mu_{0}$, convergence (i.e. $\theta<0$ ) whenever $\mu_{1}<E\left(\frac{d_{1}}{d_{0}}\right) \mu_{0}$, otherwise the profiles will be parallel.

This condition for convergence can be re-expressed as

$$
\begin{gathered}
E\left(\frac{d_{1}}{d_{0}}\right) \mu_{0}-\mu_{1}>0 \\
E\left(\frac{d_{1}}{d_{0}}\right) \frac{\mu_{0}}{\mu_{1}}-1>0 \\
E\left(\frac{s_{1}}{s_{0}}\right)-1>0
\end{gathered}
$$

So we can express these conditions as:

Convergence $(\theta<0) \Longleftrightarrow 0<E\left[\frac{s_{1}-s_{0}}{s_{0}}\right]$

Divergence $(\theta>0) \Longleftrightarrow 0>E\left[\frac{s_{1}-s_{0}}{s_{0}}\right]$

Parallel Profiles $(\theta=0) \Longleftrightarrow 0=E\left[\frac{s_{1}-s_{0}}{s_{0}}\right]$.

As we established in the proofs of Lemmas 1 and 2, and of Proposition 2 , when there is a Lorenz-improvement we can go from $s_{0}$ to $s_{1}$ through a series of transfers $h_{i j}$ and $g_{k l}$ that: 
i) appear once with a positive sign and once with a negative sign, and

ii) the sender $\mathrm{i}$ (or $\mathrm{k}$ ) is always richer than the receiver $\mathrm{j}$ (or $\mathrm{l}$ )

Hence, for each transfer $h_{i j}$ and $g_{k l}$ we have that the products

$$
\begin{aligned}
& \frac{h_{i j}}{s_{j 0}}-\frac{h_{i j}}{s_{i 0}}=h_{i j}\left(\frac{1}{s_{j 0}}-\frac{1}{s_{i 0}}\right) \\
& \frac{g_{k l}}{s_{l 0}}-\frac{g_{k l}}{s_{k 0}}=g_{k l}\left(\frac{1}{s_{l 0}}-\frac{1}{s_{k 0}}\right)
\end{aligned}
$$

are both positive.

This in turn implies that

$$
\begin{aligned}
E\left(\frac{\Delta s}{s_{0}}\right) & =\frac{1}{n} \sum_{i} \frac{s_{i 1}-s_{i 0}}{s_{i 0}} \\
& =\frac{1}{n} \sum_{i} \frac{\left(s_{i 1}-s_{i c}\right)+\left(s_{i c}-s_{i 0}\right)}{s_{i 0}} \\
& =\frac{1}{n} \sum_{i} \frac{\left(\sum_{j=1}^{n} g_{j i}-\sum_{j=1}^{n} g_{i j}\right)+\left(\sum_{j=1}^{n} h_{j i}-\sum_{j=1}^{n} h_{i j}\right)}{s_{i 0}}
\end{aligned}
$$

will be the sum of terms $h_{i j}\left(\frac{1}{s_{j 0}}-\frac{1}{s_{i 0}}\right)$ and $g_{k l}\left(\frac{1}{s_{l 0}}-\frac{1}{s_{k 0}}\right)$ for all the transfers $h_{i j}$ and $g_{k l}$. Since all these terms are non-negative, and some will be strictly positive, then the average percentage change in shares will be positive, and by our previous derivation the coefficient $\theta$ will be negative. Namely, the exact proportional change regression will be convergent.

Corollary 1. From the proof of Proposition 5 we know that $\theta<0 \Longleftrightarrow$

$$
\begin{aligned}
E\left(\frac{\Delta s}{s_{0}}\right) & =\frac{1}{n} \sum_{i} \frac{\left(s_{i 1}-s_{i c}\right)+\left(s_{i c}-s_{i 0}\right)}{s_{i 0}} \\
& =\frac{1}{n} \sum_{i} \frac{\left(\sum_{j=1}^{n} g_{j i}-\sum_{j=1}^{n} g_{i j}\right)+\left(\sum_{j=1}^{n} h_{j i}-\sum_{j=1}^{n} h_{i j}\right)}{s_{i 0}}
\end{aligned}
$$

is positive. The terms $h_{i j}\left(1 / s_{j 0}-1 / s_{i 0}\right)$ representing the transfers to go from $\mathbf{s}_{\mathbf{0}}$ to $\mathbf{s}_{\mathbf{c}}$ can be positive in the case of a Lorenz-improvement (the receiver $\mathrm{j}$ is poorer than the sender), or negative in the case of a Lorenz-worsening (the opposite case). 
The terms $g_{k l}\left(1 / s_{l 0}-1 / s_{k 0}\right)$ representing the transfers to go from $\mathbf{s}_{\mathbf{c}}$ to $\mathbf{s}_{\mathbf{1}}$ are always positive because in the case of a positional swap the receiver 1 is always poorer than the sender $\mathrm{k}$.

Hence, we can have both rising inequality (i.e. Lorenz-worsening) and convergent proportional changes as long as

$$
\frac{1}{n} \sum_{i} \frac{s_{i 1}-s_{i c}}{s_{i 0}}>\left|\frac{1}{n} \sum_{i} \frac{s_{i c}-s_{i 0}}{s_{i 0}}\right|
$$

Proposition 6. By definition

$$
r_{l}=\frac{\operatorname{cov}\left(d_{1}, d_{0}\right)}{\sqrt{V\left(d_{1}\right)} \sqrt{V\left(d_{0}\right)}}
$$

and

$$
\beta_{d}=r_{l} \frac{\sqrt{V\left(d_{1}\right)}}{\sqrt{V\left(d_{0}\right)}} .
$$

However,

$$
\begin{aligned}
\frac{\sqrt{V\left(d_{1}\right)}}{\sqrt{V\left(d_{0}\right)}} & =\frac{\sqrt{V\left(d_{1}\right)} / \mu_{1}}{\sqrt{V\left(d_{0}\right)} / \mu_{0}} \frac{\mu_{1}}{\mu_{0}} \\
& =\frac{C V\left(d_{1}\right)}{C V\left(d_{0}\right)} \frac{\mu_{1}}{\mu_{0}} .
\end{aligned}
$$

Moreover,

$$
\mu_{1}=(1+g) \mu_{0}
$$

where $g$ is the economy-wide income growth rate. Combining these equations together we obtain equation (11).

Proposition 7. First express the regression (12) in its final-on-initial form

$$
y_{1}=\alpha_{y}+\left(\delta_{y}+1\right) y_{0}+u_{y}
$$

Take the variance of both sides

$$
V\left(y_{1}\right)=\left(\delta_{y}+1\right)^{2} V\left(y_{0}\right)+V\left(u_{y}\right) .
$$


Note we can rewrite the change in variances of $y$ as

$$
\Delta V(y)=V\left(y_{1}\right)-V\left(y_{0}\right)=\delta_{y}\left(\delta_{y}+2\right) V\left(y_{0}\right)+V\left(u_{y}\right) .
$$

If the left-hand side of the equation is negative then it must be the case that $-2<\delta_{y}<0$.

Corollary 3. Consider the identity

$$
y_{1}=y_{0}+\Delta y \text {. }
$$

Taking variances on both sides of the equation we obtain

$$
V\left(y_{1}\right)=V\left(y_{0}\right)+V(\Delta y)+2 \operatorname{cov}\left(\Delta y, y_{0}\right),
$$

or simply

$$
\Delta V(y)=V(\Delta y)+2 \operatorname{cov}\left(\Delta y, y_{0}\right) .
$$

If $0<\Delta V(y)<V(\Delta y)$, then $\operatorname{cov}\left(\Delta y, y_{0}\right)<0$. Since this covariance determines the sign of $\delta_{y}$, the corollary follows.

Proposition 9. Note first that the $C V^{2}=V(s)$, since

$$
\begin{aligned}
V(s) & =V\left(\frac{d}{\mu}\right) \\
& =\frac{1}{\mu^{2}} V(d) \\
& =C V^{2} .
\end{aligned}
$$

The result in Proposition 9 follows immediately by noting that by Proposition 7 a falling $\mathrm{CV}$ implies convergence in shares $\delta_{s}<0$. Also by Proposition 8 a falling $\mathrm{CV}$ together with the specified type of Lorenz-crossing leads to a reduction in $I_{T S}()$. 